\title{
Biocompatibility and Toxicity of Nanobiomaterials
}

\author{
Xiaoming Li, ${ }^{1}$ Sang Cheon Lee, ${ }^{2}$ Shuming Zhang, ${ }^{3}$ and Tsukasa Akasaka ${ }^{4}$ \\ ${ }^{1}$ Key Laboratory for Biomechanics and Mechanobiology of Ministry of Education, School of Biological Science and Medical Engineering, \\ Beihang University, Beijing 100191, China \\ ${ }^{2}$ Department of Maxillofacial Biomedical Engineering, School of Dentistry, Kyung Hee University, Seoul 130-701, Republic of Korea \\ ${ }^{3}$ Department of Materials Science and Engineering, Johns Hopkins University, Baltimore, MD 21218, USA \\ ${ }^{4}$ Department of Biomedical Materials and Engineering, Hokkaido University, Sapporo 060-8586, Japan
}

Correspondence should be addressed to Xiaoming Li, x.m.li@hotmail.com

Received 28 November 2012; Accepted 28 November 2012

Copyright (C) 2012 Xiaoming Li et al. This is an open access article distributed under the Creative Commons Attribution License, which permits unrestricted use, distribution, and reproduction in any medium, provided the original work is properly cited.

The nanodimensionality of nature has logically given rise to the interest in using nanomaterials in biomedical field [1-3]. Over the past decades, nanobiomaterials have played significant roles in the fields of biomedical engineering and pharmaceutics. The wide variety of nanobiomaterials has encouraged their use in applications including drug delivery systems, imaging systems for diagnosis, tissue engineering, and dental/bone implant. For successful clinical applications, the investigations into biocompatibility and toxicity of nanobiomaterials are research interests of great significances. The same unique physical and chemical properties that may be beneficial in nanobiomaterials may be associated with potentially deleterious effects on human health. For example, nanobiomaterials designed for drug delivery deliberately overcome biological barriers or they may cross them unintentionally as a result of environmental exposure. Once they have been taken up, they can potentially be deposited in any region of the body. Due to their small size or specific functionalization, nanobiomaterials may cross important biological barriers and cause toxicity to very sensitive systems such as the brain or the developing fetus. Nanobiomaterials can be transported through the blood and accumulate in secondary target tissues and organs such as liver, spleen, kidney, placenta, the cardiovascular system, and central nervous system (CNS), where they may cause adverse effects [4-6]. But on the other hand, some nanobiomaterials have been found to be able to concentrate more proteins including specific proteins and these proteins might not only improve cell attachment and proliferation but also differentiate the inducible cells, which promote the tissue repair. Therefore, these nanobiomaterials might have the ability to modulate downstream stem cellular response, without exogenous growth factors, coatings, or complex ligand incorporation, and have the potential to greatly facilitate the development of tissue engineering and cellular therapies [7-9].

Biocompatibility and toxicity of nanobiomaterials are tested at three levels. First, tests are performed in vitro in cell or tissue cultures. If these tests are shown promising, further testing is performed in vivo in animal models before clinical studies are performed on humans. Existing regulatory frameworks in principle cover all important aspects; however, different behavior of nanobiomaterials from the corresponding bulk material suggests the need for specific nanopolicies or adaptation of existing frames. Unfortunately, there is still lack of validated analytical and biological methods as well as certified reference standards for exposure and hazard assessment for establishing safe doses of nanobiomaterials. Well-characterized and defined nanobiomaterials to be used as certified reference standards need to be developed as quality controls additional to standardized test protocols. Furthermore, the underlying mechanisms, for example, oxidative stress, immunotoxicity, and genotoxicity, need to be investigated and possible adverse effects should be considered.

To date, many trials have been performed to estimate in vitro and in vivo biocompatibility and toxicity of nanobiomaterials. Based on such preclinical evaluation of biocompatibility and toxicity, current research trends are focused on whether the nanobiomaterials induce toxic effects in human body. For example, some nanomaterials may be uptaken by phagocytic cells of the RES (reticuloendothelial system), to induce the production of reactive oxygen species 
and cause oxidative stress or preinflammatory cytotoxic activity in the lungs, liver, heart, brain, and so forth.

In this special issue, a dozen of articles are devoted to interaction between nanobiomaterials and cell/tissue, effect of size, shape, and surface of nanobiomaterials on their biocompatibility and toxicity, immunogenicity of nanobiomaterials, design of novel biocompatible and biodegradable nanobiomaterials, instrumentation and technology to analyze toxicity of nanobiomaterials, and challenges to overcome the toxicity of nanobiomaterials. The readers can find in this special issue not only accurate data and updated reviews on the above-mentioned aspects but also the possibility to understand accurately how nanobiomaterials interact with organs, tissues, and cells, and what is their bioavailability and biopersistence, and self-assembly mechanism of natural tissues in order to better biomimetically prepare tissue repair nanobiomaterials, to find out how the biocompatibility and toxicity of nanobiomaterials depend on their properties such as dimensions, size, shape, chemical composition, surface chemistry, and coatings, and to realize the necessity to chemically functionalize nanobiomaterials or introduce various molecules, growth factors, antigens, and specific DNA sequences, which can subsequently have positive effect on the desired cellular functions or therapeutic efficacy and to develop standard toxicological tests for nanobiomaterials so that one set of experiments can be compared with another.

\section{Xiaoming $\mathrm{Li}$ \\ Sang Cheon Lee \\ Shuming Zhang \\ Tsukasa Akasaka}

phosphate ceramics," Journal of Biomedical Materials Research $B$, vol. 97, no. 1, pp. 10-19, 2011.

\section{References}

[1] X. Li, Q. Feng, X. Liu, W. Dong, and F. Cui, "Collagen-based implants reinforced by chitin fibres in a goat shank bone defect model," Biomaterials, vol. 27, no. 9, pp. 1917-1923, 2006.

[2] X. Li, H. Gao, M. Uo et al., "Effect of carbon nanotubes on cellular functions in vitro," Journal of Biomedical Materials Research A, vol. 91, no. 1, pp. 132-139, 2009.

[3] X. Li, H. Gao, M. Uo et al., "Maturation of osteoblast-like SaoS2 induced by carbon nanotubes," Biomedical Materials, vol. 4, no. 1, Article ID 015005, 2009.

[4] X. Li, X. Liu, J. Huang, Y. Fan, and F.-Z. Cui, "Biomedical investigation of CNT based coatings," Surface and Coatings Technology, vol. 206, no. 4, pp. 759-766, 2011.

[5] X. Li, Y. Fan, and F. Watari, "Current investigations into carbon nanotubes for biomedical application," Biomedical Materials, vol. 5, no. 2, Article ID 022001, 12 pages, 2010.

[6] M. Dusinska, Z. Magdolenova, and L. Fjellsbo, "Toxicological aspects for nanomaterial in humans," Methods in Molecular Biology, vol. 948, pp. 1-12, 2013.

[7] X. Li, C. A. van Blitterswijk, Q. Feng, F. Cui, and F. Watari, "The effect of calcium phosphate microstructure on bone-related cells in vitro," Biomaterials, vol. 29, no. 23, pp. 3306-3316, 2008.

[8] X. Li, H. Liu, X. Niu et al., "The use of carbon nanotubes to induce osteogenic differentiation of human adipose-derived MSCs in vitro and ectopic bone formation in vivo," Biomaterials, vol. 33, no. 19, pp. 4818-4827, 2012.

[9] X. Li, H. Liu, X. Niu et al., "Osteogenic differentiation of human adipose-derived stem cells induced by osteoinductive calcium 

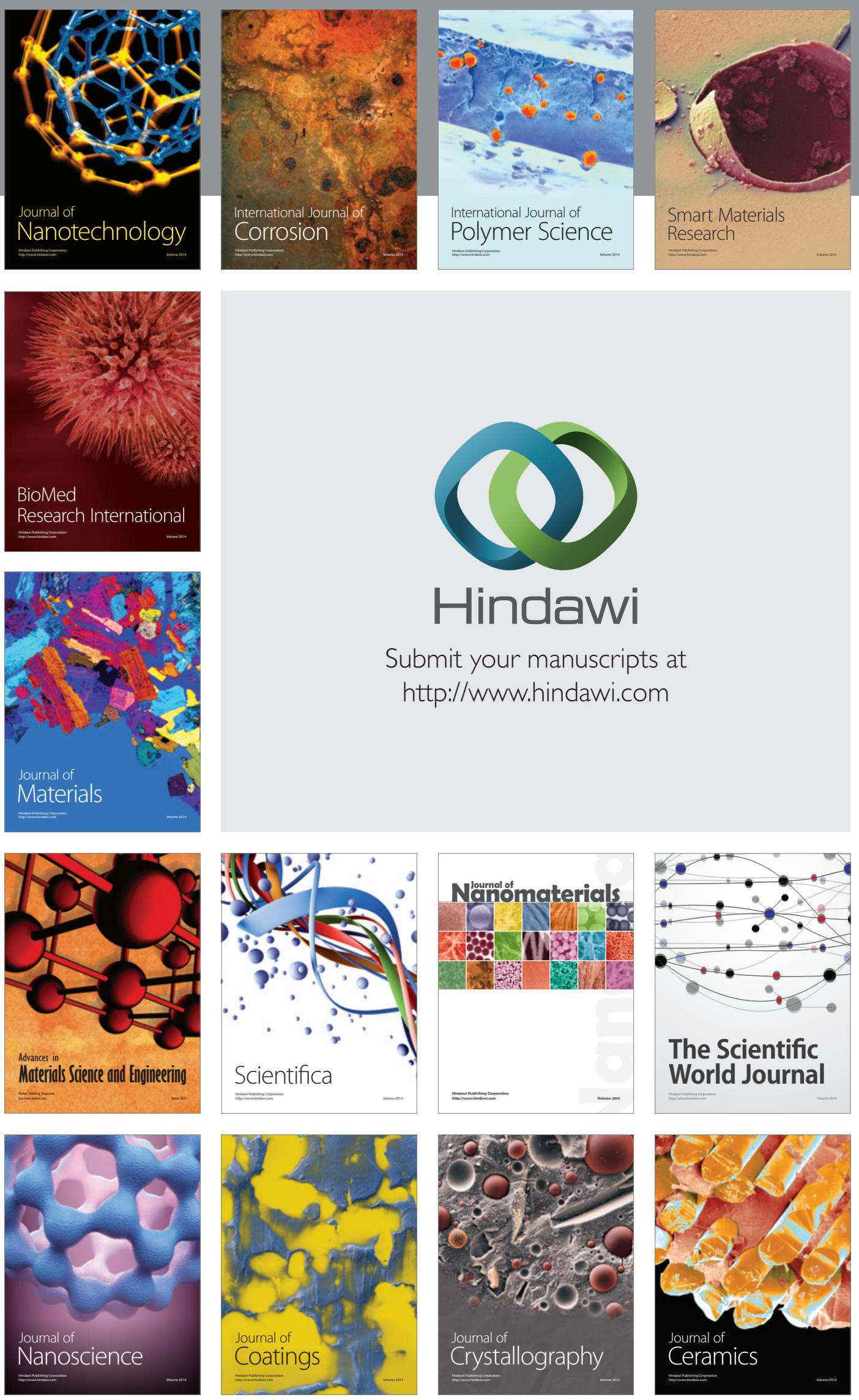

The Scientific World Journal

Submit your manuscripts at

http://www.hindawi.com

\section{World Journal}

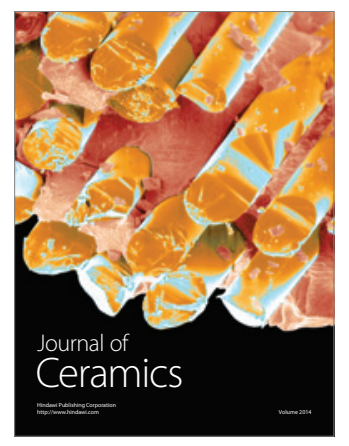

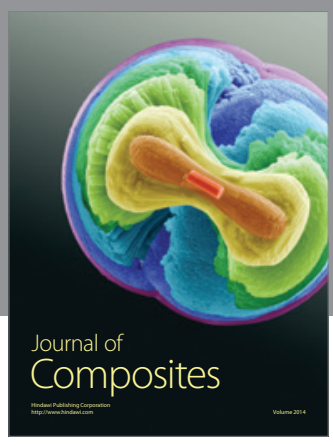
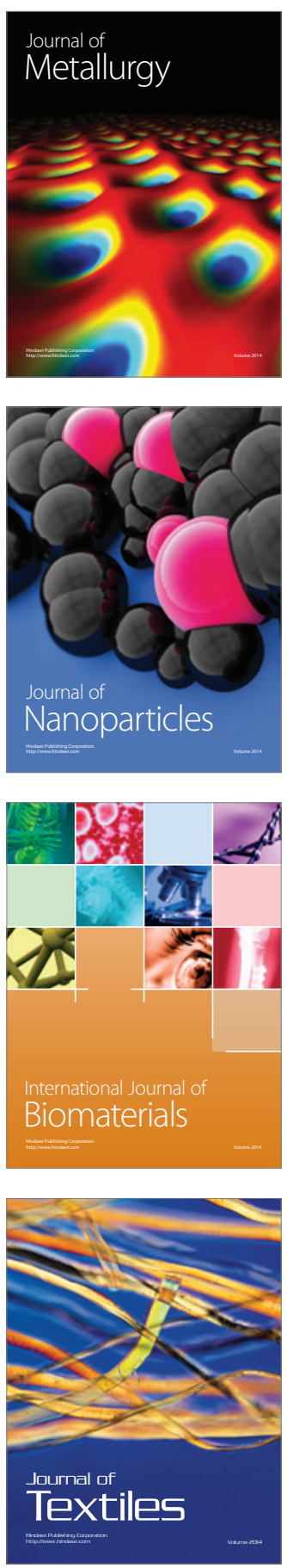